\title{
Programmable liquid crystal waveplate polarization gratings as elements for polarimetric and interference applications
}

Ignacio Moreno $^{1}$ and Jeffrey A. Davis ${ }^{2}$

${ }^{1}$ Departamento de Ciencia de Materiales, Optica y Tecnología Electrónica. Universidad Miguel Hernández, Elche, Spain

${ }^{2}$ Department of Physics, San Diego State University, San Diego CA, USA

\begin{abstract}
We review the use of a parallel aligned nematic liquid crystal spatial light modulator as a very useful and flexible device for polarimetric and interferometric applications. The device acts as a programmable pixelated waveplate, and the encoding of a linear grating permits its use as a polarization beam splitter. When a grating with a reduced period is encoded, the diffracted beams are spatially separated and the device can be used for polarimetric analysis. On the contrary when a large period grating is displayed, the beams are not spatially separated, and they are useful to realize a common path interferometric system with polarization sensitivity. The flexibility offered by the programmability of the display allows non-conventional uses, including the analysis of light beams with structured spatial polarizations.
\end{abstract}

\section{Introduction}

Polarization diffraction gratings (PDG) are diffraction gratings based on creating a periodic spatial change of the state of polarization of an incident beam of light. One simple example is a binary polarization grating, where two strips of different polarization devices constitute the basic period of the grating. Some initial efforts concentrated in the study and characterization of the properties of these gratings [1], but was Gori's proposal [2] of a PDG constructed from a series of rotating polarizers with continuous polarization gratings, that led to the identification of its potential use as elements for polarimetric analysis. After that, different PDG designs have been proposed [3,4].

Their experimental realization can be done in two ways: 1) by means of polarization subwavelength gratings with spatial variation [5], or 2) by means of liquid crystal spatial light modulator (LCSLM). Here we review our work devoted to the realization of PDG with a parallel aligned liquid crystal display (PAL-LCSLM), including examples useful for polarimetry and interferometry.

\section{Device characteristics}

Experimental results were obtained using a PAL-LCSLM [6] manufactured by Seiko Epson and having high resolution (640x480 pixels) on a 1.3" diagonal display. The pixel spacing is 42 microns and the thickness of the pixels is 4.5 microns. The PAL-LCSLM acts as an electrically controllable wave plate with a voltage dependent phase shift $\delta(\mathrm{V})$ that exceeds a range of $2 \pi$ radians as a function of gray level using the argon laser wavelength of $514 \mathrm{~nm}$.

\section{Blazed Waveplate PDGs}

Next we present some PDG results based on the use of the above described display when a linear blaze grating is displayed. Our first PDG design was a spatially variable wave plate [7], which we later operated in reflection to generate an optical triplicator [8]. Other examples of binary polarization waveplate gratings were later developed in $[9,10]$. Figure 1 shows the optical setup, which is a simple Fourier transfomer based on illuminating the display with a collimated beam and observing the pattern in the back focal plane of a converging lens.
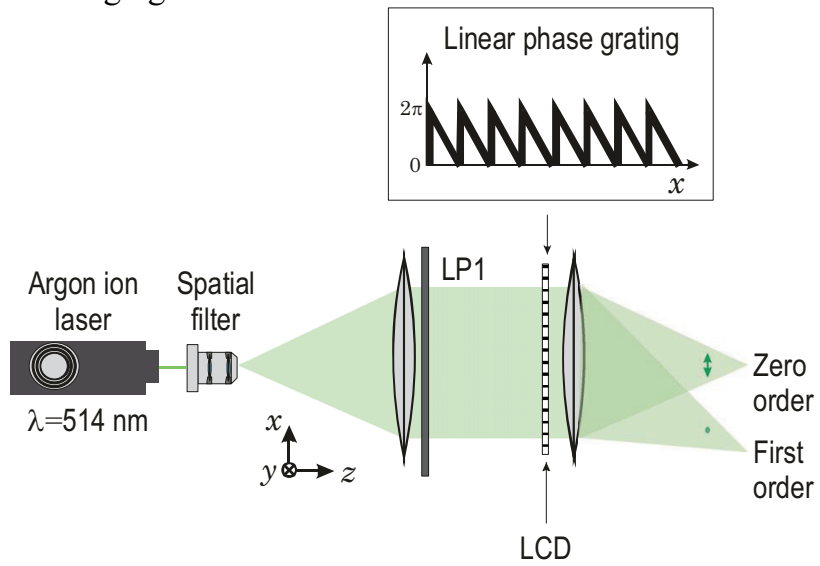

Fig. 1. Difractometer optical setup. A blazed phase grating is displayed onto the PAL-LCSLM.

This is an Open Access article distributed under the terms of the Creative Commons Attribution-Noncommercial License 3.0, which permits unrestricted use, distribution, and reproduction in any noncommercial medium, provided the original work is properly cited. 


\subsection{Linear Polarization Beam Splitter PDG}

In the original blaze waveplate grating [7], the relative phase retardation varies linearly along the $x$ direction as $\varphi(x)=2 \pi x / D$ where $D$ is the grating's period. The resulting Jones matrix of this polarization grating $\mathbf{G}(x, y)$ can be decomposed as:

$$
\begin{aligned}
\mathbf{G}(x, y) & =\left(\begin{array}{cc}
1 & 0 \\
0 & e^{2 \pi i x / D}
\end{array}\right)= \\
& =\left(\begin{array}{ll}
1 & 0 \\
0 & 0
\end{array}\right)+e^{2 \pi i x / D}\left(\begin{array}{ll}
0 & 0 \\
0 & 1
\end{array}\right)= \\
& =\mathbf{P}_{0}+e^{2 \pi i x / D} \mathbf{P}_{90} .
\end{aligned}
$$

Here $\mathbf{P}_{0}$ and $\mathbf{P}_{90}$ denote the Jones matrices of linear polarizers oriented horizontally and vertically respectively. The first term in Eq. (1) forms the zero diffraction order of the resulting diffraction pattern, and it polarizes light in the $x$ direction. The second term will form the first diffraction order, and it polarizes light in the $y$ direction. In this case, the diffraction pattern only consists of two orders and the energy in each order is proportional to the power carried by the $x$ and $y$ linearly polarized components of the incoming light. Thus the polarization grating effectively splits light in two orthogonal polarization states.

The output electric field vector behind the LCSLM is given by the product of this set of matrices with the input electric field vector given by

$\mathbf{E}=\left(\begin{array}{c}E_{x} \\ E_{y} e^{i \phi}\end{array}\right)$.

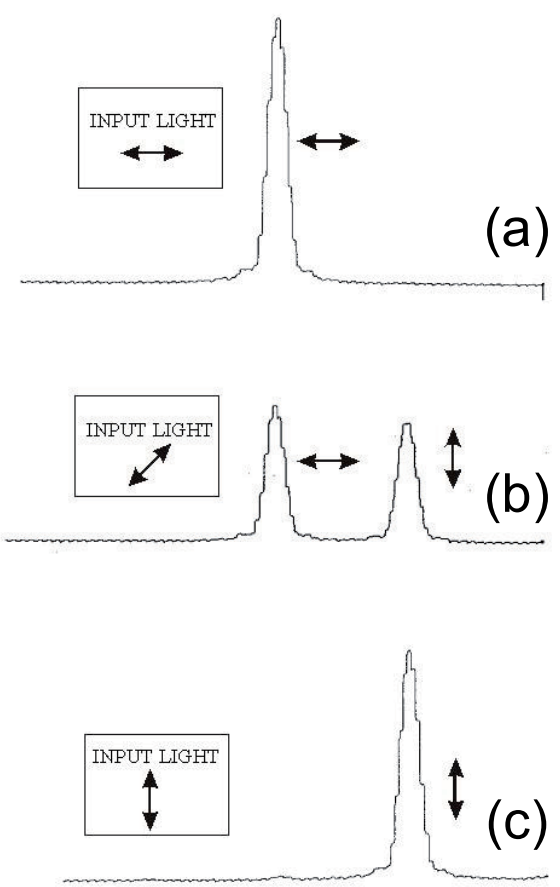

Fig. 2. Experimental results for grating separating linearly polarized components of the incident light. Input light linearly polarized (a) perpendicular, (b) at $45^{\circ}$ and (c) parallel to director axis.
The intensity diffracted into the zero and first diffraction orders is given by

$\eta_{0}=\left|E_{x}\right|^{2}, \eta_{1}=\left|E_{y}\right|^{2}$.

The experimental results are shown in Fig. 2. In Fig. 2(a), the input light is polarized perpendicular to the director axis of the LCD (which is oriented in the vertical direction) and only the zero order is formed. When the input light is polarized at $45^{\circ}$ in Fig. 2(b), the input light can be decomposed into horizontal and perpendicular components, each of which forms its respective diffraction pattern. Finally when the input light is polarized along the director axis in Fig. 2(c), only the first diffracted order is formed.

\subsection{Circular Polarization Beam Splitter PDG}

A simple modification of this grating allows the evaluation of the components of light in another orthonormal basis. If the grating given in Eq. (1) is sandwiched between two quarter-waveplates (QWP) with their principle axes oriented at $+45^{\circ}$ and $-45^{\circ}$, the resulting grating is given by:

$$
\begin{aligned}
\mathbf{G}(x) & =\mathbf{W}_{\lambda / 4}\left(45^{\mathrm{o}}\right) \cdot\left(\begin{array}{cc}
1 & 0 \\
0 & e^{2 \pi i x / D}
\end{array}\right) \cdot \mathbf{W}_{\lambda / 4}\left(45^{\mathrm{o}}\right)= \\
& =\frac{1}{2}\left(\begin{array}{cc}
i & -1 \\
1 & i
\end{array}\right)+e^{2 \pi i x / D} \frac{1}{2}\left(\begin{array}{cc}
i & 1 \\
-1 & i
\end{array}\right)= \\
& =i\left(\mathbf{P}_{\mathbf{L}}+e^{i \phi} \mathbf{P}_{\mathbf{R}}\right) .
\end{aligned}
$$

Here $\mathbf{W}_{\lambda / 4}(\theta)$ stands for the Jones matrix of the QWP oriented at angle $\theta . \mathbf{P}_{\mathbf{L}}$ and $\mathbf{P}_{\mathbf{R}}$ denote the Jones matrices for right-handed $(\mathrm{R})$ and left-handed (L) circular polarizers. For this case, the light that is diffracted in the zero order is the right-circularly polarized component of the incoming light while the first order carries the leftcircularly polarized component.

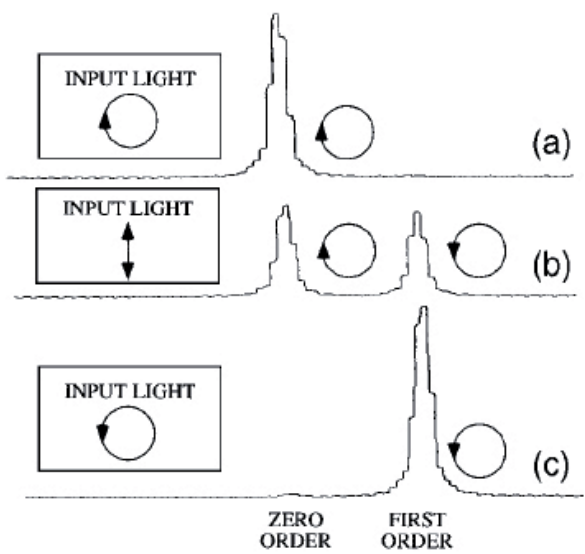

Fig. 3 Experimental results for grating separating circularly polarized components of the incident light. Input light (a) R circularly polarized, (b) linearly polarized, and (c) L circularly polarized. 
Experimental results are shown in Fig. 3. In Fig. 3(a), the input light is right hand circularly polarized and only the zero order is formed. In Fig. 3(b), the light is polarized parallel to the director axis and can be decomposed into equal amounts of left and right hand circularly polarized light. Consequently, both diffracted orders are formed. Finally the input light is left circularly polarized and only the +1 diffracted order appears.

Therefore, these two PDG designs have been characterized by the production of two diffraction orders, where the two polarization states of the light are orthogonal (linearly or circularly polarized). These designs can be used to perform Stokes polarimetry of an incident light beam.

\section{Common Path PDG based Polarization Interferometer}

The above system can be easily converted into a polarization sensitive common path interferometer by addressing a grating with a very low frequency [11]. Figure 4 shows the optical setup. We illuminate the LCSLM with linearly polarized light oriented at $\pm 45^{\circ}$ with respect to the LC director, which is vertically oriented. We encode blazed gratings that have a large period. The horizontally polarized component of the incident beam is not affected by the grating, while the vertical component is diffracted at a small angle that is proportional to the period of the grating. Consequently the two beams overlap. Because the two beams have orthogonal polarizations, they do not interfere unless an analyzer is in included in the common way. Because of the pixel structure of the SLM, additional diffracted orders are produced with relative angles of approximately $12.5 \mathrm{mrad}$. As the output plane is moved farther from the SLM, these patterns diverge and can create more complicated interference patterns. However, we find it easier to operate at close distances (within $15 \mathrm{~cm}$ ) so that these patterns are coincident. This results in a compact polarization interferometer that can be used to measure phase-shifts between the orthogonal polarizations. We record the interference patterns with a CCD camera.

Figure 5 shows the output pattern as the analyzer is rotated. For this experiment, the input polarization is linear at $+45^{\circ}$, and the grating has a period of 64 pixels (causing a deflection of only $0.19 \mathrm{mrad}$ ). For this experiment, the blazed grating lines are oriented parallel to the LC director (vertical orientation) and the diffracted beam is diffracted horizontally. Figure 5 shows the vertical fringe pattern caused by the interference by the undiffracted beam (zero order) and the first diffracted order. Figs. 5(a) and 5(c) show the fringes when the analyzer polarizer is oriented at $\pm 45^{\circ}$. Note the relative half period displacement between the two figures due to the additional $\pi$ phase shift that occurs when the analyzer is rotated by $90^{\circ}$. When the analyzer is aligned in either the horizontal direction in Fig. 5(b) or the vertical direction (not shown), only one beam is transmitted, and the pattern is uniform.
The common path interferometer can be used to visualize phase patterns. As an example, we consider optical vortices, obtained by adding the helical phase characteristic of an optical vortex to the grating pattern. This phase varies azimuthally as $\exp (\operatorname{im} \theta)$, where $m$ is an integer number known as the topological charge, and $\theta$ is the azimuthal angle [12]. Figure 6 shows the results for $m=2,3$, and 4 where we again used a grating period of 64 pixels, as in Fig. 5, and illustrates some interesting features. In doing these experiments, we introduced the Soleil Babinet compensator in order to correct for the absolute phase differences provided between the extraordinary and ordinary axes of the LCSLM. This allowed us to make the vortex interference patterns symmetric. The result is an interference fringe pattern that breaks at the vortex, and the number of interference fringes increases in proportion to the value of the topological charge.

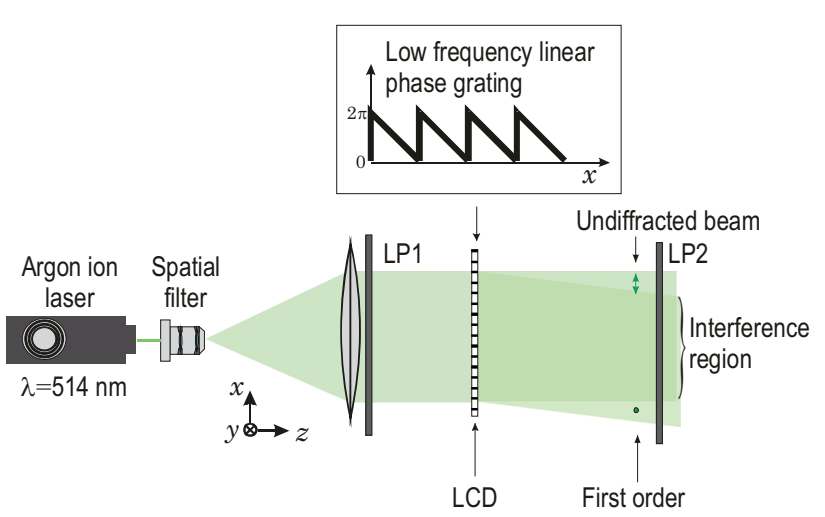

Fig. 4. Common path polarization interferometer optical setup.

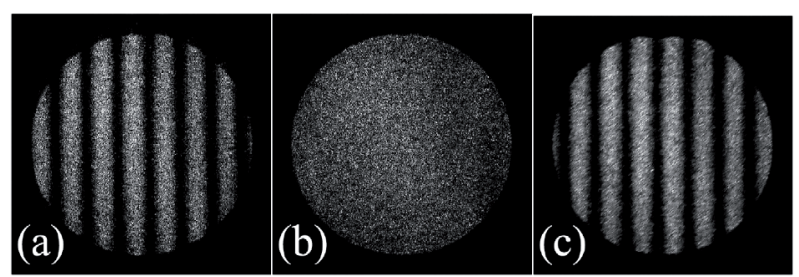

Fig. 5. Optical interference pattern as the analyzer is oriented at angles of (a) $-45^{\circ}$, (b) vertical, and (c) $+45^{\circ}$.

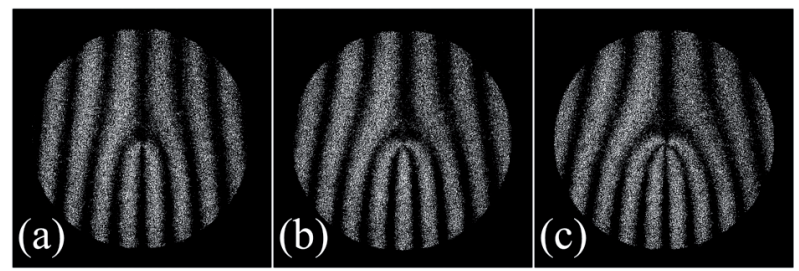

Fig. 6. Self interference pattern when helical phase patterns $\exp (\operatorname{im} \theta)$ with different topological orders $m$ are added to the blazed grating: (a) $m=+2$, (b) $m=+3$, (c) $m=+4$. 
The technique can be extended to circular polarization analysis, by adding the two QWP in the same manner as in Fig. 4. Then the reference beam is characterized by one sense of circularly polarized light, while the object beam has the opposite sense of circular polarization. Thus the proposed system acts as a circular polarization splitting common path interferometer [13] and is sensitive to phase shifts between L and R circularly polarized beams, which correspond to the angle of rotation in polarization rotators.

In order to test this system, we included an optically active medium between the optical rotator system and the final polarizer. Optical activity is characterized by a different index of refraction for left- and right-circular polarized light and can be regarded as having a phaseshift between circular polarization states. Materials showing optical activity, when illuminated with linearly polarized light, rotate the plane of polarization by an angle $\theta$ that depends on the thickness of the medium and can be either positive or negative. The phase shift between the circular polarization states is $2 \theta$. The proposed interferometer presents the unique feature that visualizes optical activity as a displacement of the fringes (in opposition to regular interferometers, where optical activity results in a reduction of fringe visibility caused by the different polarization orientations).

Figures 7 and 8 show the results when an active medium is inserted between the second QWP and the final analyzer polarizer. For comparison, Figs. 7(a) and 8(a) show the interference pattern without the active medium. Figures 7(b) and 8(c) show the cases where a sucrose sugar $\left(\mathrm{C}_{12} \mathrm{H}_{22} \mathrm{O}_{11}\right)$ solution of commercially available Karo $^{\circledR}$ Light Corn Syrup with cell thicknesses of $1 \mathrm{~cm}$ and $2 \mathrm{~cm}$ are inserted into the left half of the system. The dark region in the center of the figures shows the wall of the optical cell. Our sample has a rotation of $+18 \mathrm{deg} / \mathrm{cm}$ at the $514.5 \mathrm{~nm}$ wavelength. The interference fringes on the left side clearly move downwards compared with Fig. 7(a), and the displacement is greater for the thicker active cell. We again draw the horizontal lines to clearly see the movement of the fringes. The fringe displacements of (b) roughly 0.1 fringe and (c) 0.2 fringe agree with theory.

Figure 8 shows results using a similar optical cell containing a supersaturated solution of fructose sugar $\left(\mathrm{C}_{6} \mathrm{H}_{12} \mathrm{O}_{6}\right)$. This material is characterized by the opposite sense of optical activity and we present the same set of experiments. Figure 8(a) is identical to Fig. 7(a), while Figs. 8(b) and 8(c) show the results when cell thicknesses of $1 \mathrm{~cm}$ and $2 \mathrm{~cm}$ are inserted into the left half of the system. Again the dark region in the center of the figures shows the wall of the optical cell. Our sample has a rotation of $-15 \mathrm{deg} / \mathrm{cm}$ at the $514.5 \mathrm{~nm}$ wavelength (note that these rotation values depend on the concentration of the sugar). The interference fringes on the left side now move in the opposite direction by smaller amounts compared with Fig 7, but in agreement with theory. In all cases, the magnitudes and directions of the fringe shifts agree with theory.
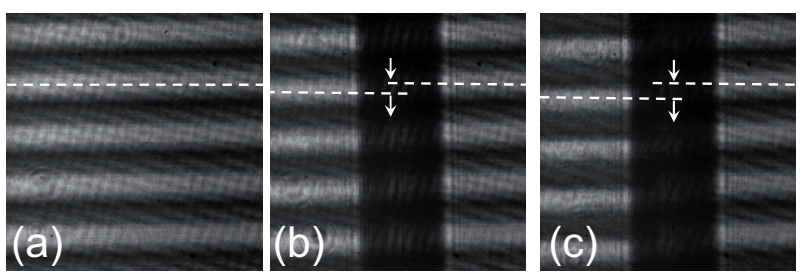

Fig. 7. Interference patterns: (a) without active media, (b)-(c) with sucrose sugar solution introduced in the left part, $1 \mathrm{~cm}$ and $2 \mathrm{~cm}$ thick respectively.
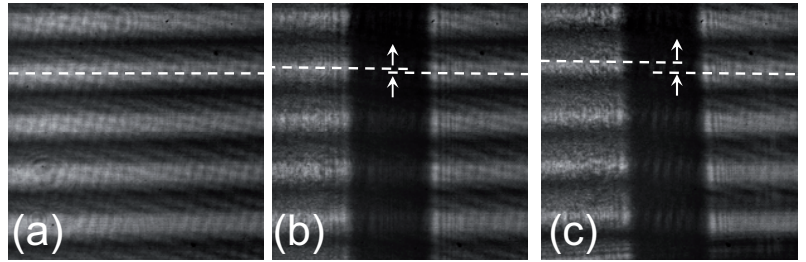

Fig. 8. Interference patterns: (a) without active media, (b)-(c) with fructose sugar solution introduced in the left part, $1 \mathrm{~cm}$ and $2 \mathrm{~cm}$ thick respectively.

\section{Conclusions}

In summary, we presented the use of a parallel aligned nematic liquid crystal spatial light modulator as a very useful and flexible device for polarimetric and interferometric applications. The device acts as a programmable pixelated waveplate, and the encoding of a linear grating permits its use as a polarization beam splitter. When a grating with a reduced period is encoded, the diffracted beams are spatially separated and the device can be used for polarimetric analysis.

On the contrary when a large period grating is displayed, the beams are not spatially separated, and they are useful to realize a common path interferometric system with polarization sensitivity. The flexibility offered by the programmability of the display allows nonconventional uses, which might include the analysis of light beams with structured spatial polarizations

\section{References}

1. M. Le Docen, P. Pellat-Finet, Opt. Comm. 151, 321 (1998).

2. F. Gori, Opt. Lett. 24, 584 (1999).

3. J. Tervo, J. Turunen, Opt. Lett. 25, 785 (2000).

4. G. Cincotti, IEEE J. Quant. Electron. 39, 1645-1652 (2003).

5. Z. Bomzom, V. Kleiner, E. Hasman, Opt. Lett. 26, 33 (2001).

6. J. A. Davis, P. Tsai, D. M. Cottrell, T. Sonehara, J. Amako, Opt. Eng. 38, 1051 (1999).

7. J. A. Davis, J. Adachi, C. R. Fernández-Pousa, I. Moreno, Opt. Lett. 26, 587 (2001).

8. C. R. Fernández-Pousa, I. Moreno, J. A. Davis, J. Adachi, Opt. Lett. 26, 1651 (2001).

9. J. A. Davis, G. H. Evans, Opt Lett. 29, 1443-1445 (2004). 
10. A. Martínez-García, I. Moreno, M. M. SánchezLópez, P. García-Martínez, Appl. Opt. 48, 2903 (2009).

11. I. Moreno, J. A. Davis, F. A. Klein, M. J. Mitra, Appl. Opt. 47, 1797 (2008).

12. K. Crabtree, J. A. Davis, I. Moreno, Appl. Opt. 43, 1360 (2004).

13. J. A. Davis, B. M. L. Pascoguin, I. Moreno, A. NavaVega, Opt. Lett. 34, 1486 (2009). 